\title{
Prática centrada no cliente na reabilitação: definição, instrumentos e desafios
}

\section{Client-centered practice in rehabilitation: definition, instruments and challenges}

\author{
Daniela Virgínia Vaz ${ }^{1}$, Luísa Graziella Jubilini², Letícia Costa Queiroz ${ }^{2}$
}

http://dx.doi.org/10.11606/issn.2238-6149.v28i1p122-127

Vaz DV, Jubilini LG, Queiroz LC. Prática centrada no cliente na reabilitação: definição, instrumentos e desafios. Rev Ter Ocup Univ São Paulo. 2017 jan.-abr.;28(1):122-7.

RESUMO: Introdução: $\mathrm{Na}$ reabilitação, a habilidade do paciente em lidar com a sua condição de saúde e tomar decisões, sua motivação e sua adesão, são determinantes para os desfechos terapêuticos. A prática centrada no cliente (PCC) reconhece e promove a centralidade dos interesses de pacientes e por isso seu conhecimento é fundamental para o avanço da reabilitação. Objetivos: i) identificar os fundamentos da PCC, ii) descrever instrumentos para sua implementação, iii) registrar resultados da PCC, e $i v$ ) analisar as principais dificuldades e benefícios da PCC. Método: Revisão narrativa a partir de bancos de dados e cruzamento de referências. Resultados: segundo estudos revisados, a PCC é uma abordagem clínica colaborativa e empoderadora do paciente, que reconhece-o no centro do processo decisório, aumentando a adequação do cuidado. Diferentes instrumentos simples podem ser imediatamente adotados na clínica. Ainda poucos estudos documentam resultados da PCC na reabilitação. Para implantação da PCC é necessário vencer desafios no nível individual de terapeutas e pacientes e no nível institucional de serviços de saúde.

DESCRITORES: Planejamento de assistência ao paciente; Reabilitação; Revisão.
Vaz DV, Jubilini LG, Queiroz LC. Client-centered practice in rehabilitation: definition, instruments and challenges. Rev Ter Ocup Univ São Paulo. 2017 Jan.-Apr.;28(1):122-7.

ABSTRACT: Introduction: in rehabilitation, a patient's ability cope with a health condition, make decisions, find motivation and adhere to treatment are determinants of therapeutic outcomes. Client-centered practice (CCP) recognizes and promotes patients' interests as central to care delivery. CCP is essential for the advancement of rehabilitation. Objectives: i) identify the fundamentals of CCP, ii) describe clinical instruments available for implementing it, iii) register $\mathrm{CCP}$ results obtained so far, iv) analyze the main barriers and benefits of CCP. Methods: narrative review of literature from databases and reference lists. Results: based on reviewed studies, CCP is a collaborative approach to clinical practice that aims to empower patients and recognize them at the center of the decision making process, in order to deliver better care. Several simple instruments can be immediately used in clinical practice. Few studies have investigated effects of CCP in rehabilitation. Challenges at the individual level of therapists and patients as well as at the institutional level of health services need to be met in order to implement CCP.

KEYWORDS: Patient care planning; Rehabilitation; Review.

Este trabalho foi iniciado como monografia de graduação.

1. Universidade Federal de Minas Gerais (UFMG), Departamento de Fisioterapia. Belo Horizonte, Minas Gerais, Brasil. Professora Adjunta. E-mail: danielavvaz@gmail.com

2. Universidade Federal de Minas Gerais (UFMG), Departamento de Fisioterapia, Bacharel em Fisioterapia. Belo Horizonte, Minas Gerais, Brasil. E-mail: luisajubilini@hotmail.com, leticiacq1@hotmail.com

Endereço para correspondência: Daniela Virgínia Vaz. UFMG, Departamento de Fisioterapia. Av. Pres. Antônio Carlos, 6627 Pampulha. Belo Horizonte, MG. CEP: 31270-901. 


\section{INTRODUÇÃO}

$\mathrm{D}$ urante a reabilitação, a habilidade de um paciente em lidar com sua condição de saúde e tomar decisões, sua motivação e sua adesão são determinantes para os desfechos terapêuticos. Um modelo de prática clínica que analisa estes fatores é a "prática centrada no cliente" (client-centred practice ou patient-centered care), doravante PCC. O empoderamento de pacientes e familiares para efetiva participação na definição de objetivos terapêuticos é primordial nesse processo. Acreditamos que o conhecimento da PCC é fundamental para a missão da Fisioterapia e da Terapia Ocupacional de atender as demandas dos pacientes com qualidade. Assim, esta revisão tem como objetivos: i) identificar os fundamentos da PCC, ii) descrever instrumentos utilizados na sua implementação, iii) registrar resultados documentados até o momento, e iv) analisar desafios para seu desenvolvimento.

\section{MÉTODOS DE REVISÃO}

Foram realizadas buscas nas bases do PubMed e Google Scholar com combinações dos termos "patient centered care", "client-centered practice", "physical therapy", "physiotherapy", "occupational therapy", "rehabilitation". Dos artigos resultantes, 36 títulos foram selecionados como potencialmente relevantes. A leitura dos resumos resultou em 20 trabalhos selecionados. Trabalhos relevantes indicados nas listas de referências bibliográficas também foram incluídos.

Ao longo das buscas, notou-se profusa literatura sobre PCC proveniente da Terapia Ocupacional (TO). Os princípios da PCC parecem ter sido imbuídos na formação e na cultura profissional de terapeutas ocupacionais, mais do que de quaisquer outros profissionais da reabilitação ${ }^{1}$. As evidências e análises presentes nesta literatura são muito relevantes tanto para a Terapia Ocupacional quanto para a Fisioterapia, uma vez que estas profissões frequentemente trabalham juntas e atendem populações semelhantes. As duas profissões se diferenciam em seus núcleos, mas compartilham campos. Por um lado o núcleo é a aglutinação de conhecimentos e práticas próprias de cada profissão, definidor de sua especificidade. O campo, por outro lado, seria um espaço de limites imprecisos onde cada profissão busca em outras apoio para cumprir suas tarefas teóricas e práticas $^{2}$. Assim, foram revisados trabalhos das duas áreas. A seguir, apresentamos resultados da revisão para o objetivo i): identificar os elementos fundamentais da PCC.

\section{RESULTADOS E DISCUSSÃO}

\section{Definição de PCC}

Reconhecer que cada paciente pode apresentar respostas únicas para uma mesma condição de saúde coloca o cliente, seu contexto e sua perspectiva pessoal no centro do processo terapêutico. Com base nesta centralidade, a PCC pode ser definida como uma abordagem clínica ancorada no respeito e parceria com o cliente, que reconhece a sua autonomia, a sua necessidade de fazer as próprias escolhas sobre cuidados que receber, a sua privilegiada experiência sobre sua condição de saúde, e seu potencial para contribuir para o processo terapêtico. Esta abordagem procura garantir serviços acessíveis e adequados ao contexto particular em que o cliente vive ${ }^{3,4}$. Em suma, a PCC é uma abordagem colaborativa que busca empoderar o paciente, reconhecendo-o no centro da tomada de decisões para aumentar a qualidade do processo terapêutico. Desde a avaliação, escolha de objetivos e métodos de intervenção até a documentação de resultados, os valores e preferências do paciente são determinantes.

É documentado, no entanto, um descompasso entre os valores e preferências dos profissionais, especialmente fisioterapeutas, e pacientes. Por um lado, modelos tradicionais de tratamento priorizam a remediação de deficiências de órgãos e sistemas, como a força muscular e amplitude de movimento. Por outro lado, pacientes desejam melhorar o desempenho em atividades que consideram essenciais para seu bem-estar físico, psicológico e social ${ }^{5}$. Remediar déficits pode ser um componente necessário da terapia, mas não pode ser seu fim último, pois em si é insuficiente para a promoção dos interesses dos pacientes. Na PCC, estes interesses são centrais para o processo terapêutico. Para identificálos e definir objetivos terapêuticos, assim como definir formas de acompanhar resultados, diversos instrumentos padronizados estão disponíveis. Eles são caracterizados na seção seguinte, conforme objetivo $i i$ deste trabalho.

\section{Instrumentos para implementação da PCC em reabilitação}

Os instrumentos a seguir são todos baseados em entrevistas para definir interesses e objetivos dos pacientes. Em algumas situações, o terapeuta pode considerar tais objetivos inapropriados ou irrealistas. No entanto, sendo a PCC um modelo de parceria terapeuta-paciente, deve haver abertura para discussão e colaboração. Em outras situações, o terapeuta pode considerar que a percepção do paciente 
sobre sua situação seja distorcida. Em geral, no entanto, pacientes tem ótimo discernimento sobre suas dificuldades funcionais ${ }^{6}$. Familiares ou cuidadores também podem ser entrevistados nestes casos ou no caso de pacientes bebês, crianças ou pessoas com déficits cognitivos graves 6 . Também pode ser impróprio fazer entrevistas para escolha de objetivos quando há necessidade de intervenção imediata, curta ou local, como por exemplo, mobilização pós-operatória ou higiene pulmonar.

\section{Goal Attainment Scaling (GAS)}

A GAS é bastante utilizada por combinar a parceria com o paciente para definição de objetivos e possibilidade de analisar a evolução dos resultados. Ela é usada em diversas áreas da saúde independente de diagnósticos. Sua aplicação consiste em cinco passos ${ }^{7}$. No primeiro, o paciente define suas principais limitações e seus objetivos, incluindo quaisquer atividades. Os objetivos devem ser relevantes para o paciente, compreensíveis, mensuráveis, relacionados ao desempenho de atividades funcionais e atingíveis dentro de um prazo pré-estabelecido?

No segundo, o paciente atribui níveis de importância e dificuldade para cada objetivo com escores de 0 a 3 . Zero significa nenhuma importância ou dificuldade e 3, importância ou dificuldade máximas. Caso o paciente classifique algum objetivo como 0 , esse objetivo deverá ser renegociado e substituído por outro de maior prioridade.

No terceiro, são estabelecidas expectativas de desempenho funcional após o tratamento. Estas expectativas devem ser realistas, prováveis, e escritas em linguagem mais específica possível. Assim, ao final do tratamento, haverá critérios claros e mensuráveis de desempenho que permitirão definir se a expectativa foi alcançada ou não.

No quarto, constrói-se uma escala de desempenho que varia de $-2 \mathrm{a}+2$ : o escore 0 equivale à expectativa de resultado elaborada no terceiro passo; $-1 \mathrm{e}-2$ equivalem a possíveis desfechos um pouco aquém e muito aquém da expectativa; +1 e +2 , um pouco além e muito além da expectativa. Estes níveis devem cobrir intervalos de mudança clinicamente relevantes e aproximadamente iguais. Usualmente, o nível -1 ou -2 representa o desempenho atual do paciente.

No passo final, após a classificação dos resultados obtidos depois da terapia em uma das categorias de -2 a +2 , pode ser calculado um escore total. O cálculo envolve o valor de desempenho antes e depois do tratamento, assim como os valores de dificuldade e importância.

A GAS é um instrumento válido e bastante útil para facilitar a PCC. A aplicação pode ser feita em cerca de 30 minutos. Os principais erros que comprometem sua utilização são o estabelecimento de metas não realistas, com progressão pouco significativa entre níveis e com critérios insuficientes para diferenciar um nível do outro. Exemplos práticos estão disponíveis para melhor compreensão do método ${ }^{7}$.

\section{Canadian Occupational Performance Measure}

A Medida Canadense de Desempenho Ocupacional (MCDO), conforme a tradução para o português, foi desenvolvida por Terapeutas Ocupacionais e também incorporada à atuação de Fisioterapeutas. Seu foco é a documentação da funcionalidade em três áreas de desempenho: autocuidado, produtividade e lazer ${ }^{6}$. Em uma entrevista semiestruturada o paciente identifica ocupações, atividades ou tarefas nas quais tem dificuldade nas três áreas de desempenho. $\mathrm{O}$ formulário da MCDO tem várias sugestões de atividades para ajudar o paciente a analisar de maneira abrangente as suas questões funcionais.

Após a entrevista, opcionalmente, o paciente atribui notas de 1 a 10 para a importância de cada tarefa. As cinco mais importantes são eleitas como focos da terapia. Em seguida, para estas tarefas-foco, o paciente avalia seu nível de desempenho e de satisfação com notas de 0 a 10. Os escores totais de desempenho são dados pela soma das notas de cada tarefa, com o total dividido pelo número de tarefas. O mesmo procedimento é usado para escores totais de satisfação. Novos escores podem ser calculados após a intervenção para documentação de progresso. Diferenças de dois ou mais pontos são consideradas clinicamente significativas. Diversos estudos demonstram a validade, confiabilidade e sensibilidade da $\mathrm{MCDO}^{6}$.

\section{Patient-Specific Functional Scale}

A Escala Funcional Específica do Paciente (EFEP) foi desenvolvida especialmente para pacientes com desordens musculoesqueléticas. Ela consiste em uma entrevista em que o paciente elege cinco atividades importantes cuja execução esteja dificultada ou impossibilitada devido à sua deficiência ou problema de saúde. Para cada atividade, o paciente atribui uma nota de dificuldade de 0 ("incapaz de realizar a atividade") a 10 ("capaz de realizar a atividade no mesmo nível de antes da lesão ou problema"). Em reavaliações subsequentes, o paciente repontua as atividades iniciais, ou identifica novas limitações de atividade surgidas ao longo do tempo. A EFEP possui uma excelente confiabilidade e validade ${ }^{8}$. 
Vaz DV, et al. Prática Centrada na Reabilitação. Rev Ter Ocup Univ São Paulo. 2017 jan./abr.;28(1):122-7.

\section{Patient Goal Priority List}

Este instrumento consiste em uma listagem de objetivos prioritários, desenvolvido especialmente para pacientes que sofrem com desordens musculoesqueléticas e dor persistente. Primeiramente, o paciente lista atividades (sem limitação de número) que tem mais dificuldade para realizar. Em seguida, ranqueia-as por prioridade. Depois, define com que frequência (diária, semanal ou mensal) cada atividade é feita. Por último, ordena as atividades por nível de dificuldade. As atividades-foco da terapia são aquelas 4 ou 5 atividades que atingem o topo das listas de dificuldade, prioridade e frequência. A validade e a confiabilidade desta forma de listagem não foi especificamente estabelecida ${ }^{9}$.

\section{Efeitos documentados da $\mathrm{PCC}$ em reabilitação}

Um estudo com vítimas de traumatismo cranioencefálico (TCE) mostrou que o estabelecimento de objetivos na perspectiva da PCC fornece estrutura para a reabilitação, garante foco para o trabalho, promove a motivação dos pacientes e terapeutas, e favorece satisfação com o progresso alcançado ${ }^{10}$. A PCC também favorece redução de ansiedade ${ }^{11}$, melhoria do senso de auto-eficácia e autonomia, e maior participação no processo de reabilitação ${ }^{10}$. Estes são efeitos importantes, uma vez que podem aliviar a angústia que normalmente acompanha problemas de saúde.

Há também alguma evidência de que a PCC promove ganhos no desempenho funcional. Pacientes com TCE altamente envolvidos na definição de objetivos comparados com pacientes com baixo envolvimento tiveram desempenho significativamente mais alto ao final de um programa de tratamento em $\mathrm{TO}^{12}$. Entre pacientes com doenças reumatológicas na fisioterapia, o grupo que participou de definição colaborativa de objetivos obteve maior progresso em habilidades funcionais, dor, amplitude de movimento e força muscular ${ }^{13}$. Resultados semelhantes foram encontrados para a independência funcional e autocuidado após tratamento de TO em pacientes com variadas condições de saúde ${ }^{14}$.

No entanto, outros estudos não observaram diferenças entre grupos que participaram ou não da definição colaborativa de objetivos ${ }^{15,16}$. Além disso, um estudo verificou piores resultados no nível de fadiga e qualidade de vida de pacientes com esclerose múltipla que receberam serviços de TO centrada no cliente comparada à abordagem usual $^{17}$. É possível que a mudança de processos requerida para a implantação da PCC tenha desviado tempo que teria sido dedicado ao tratamento para avaliações e reuniões com os pacientes. A questão da alocação de tempo precisa ser considerada na análise dos efeitos da PCC.

\section{Desafios para a implantação da PCC}

Barreiras para a PPC ocorrem tanto no nível individual, relativo a terapeutas e pacientes, quanto no nível institucional. $\mathrm{O}$ reconhecimento dessas barreiras é fundamental para a sua superação. A literatura fornece algumas sugestões.

Para terapeutas, muitas barreiras se relacionam a limites nas suas habilidades e competências de comunicação e negociação ${ }^{18}$. Outras barreiras são atitudinais: alguns fisioterapeutas acreditam que já fazem, ou sempre fizeram $\mathrm{PCC}^{19}$, apesar das diversas evidências de descompasso entre o que pacientes desejam e o que terapeutas oferecem, além da documentada baixa habilidade de clínicos de compartilhar decisões. Contra esta barreira se coloca o esclarecimento de que a PCC é um processo em aberto, que sempre pode ser aprimorado. Ainda, alguns terapeutas relatam que se sentem ameaçados em dividir poder com pacientes e suas famílias e temem perder prestígio e reconhecimento quando precisam aderir à $\mathrm{PCC}^{20}$.

Certamente, a adesão à $\mathrm{PCC}$ requer que o terapeuta transite da função de expert com total poder de decisão diagnóstica e terapêutica para a função de parceiro ou consultor do paciente. Alguns profissionais temem que isto implique em desvalorização de conhecimento e habilidades técnico/científicas específicas do terapeuta ocupacional ou fisioterapeuta. A PCC muitas vezes exige uma ampliação do foco de trabalho para acomodar os interesses dos pacientes para além de problemas típicos do núcleo de cada profissão, exigindo articulação entre campos de atuação, como mencionado anteriormente. Alguns profissionais expressam desconforto com esta ampliação, questionando se isso não descaracteriza sua profissão originária. Curiosamente, esta desvantagem muitas vezes é identificada pelos pacientes como uma das vantagens da $\mathrm{PCC}^{19,20}$. Esta tensão reflete questões mais amplas, históricas e teóricas relativas à evolução das identidades profissionais.

Por último, pode haver percepção de tensões entre a PCC e a prática baseada em evidências, que envolve um imperativo ético de oferecer a conduta para a qual existam as melhores evidências científicas, dadas as habilidades do profissional e as preferências do cliente. Fisioterapeutas trabalhando sob a filosofia da PCC relataram sentir certa pressão para continuar terapias que não tinham suporte em evidências, mas que eram desejadas pelas famílias, e expressaram temor de que seu trabalho pudesse levar a resultados negativos para os pacientes ${ }^{19,20}$. Este conflito se relaciona com os limites do conhecimento fornecido por estudos randomizados controlados, cuja aplicabilidade pode ser limitada em situações altamente individualizadas 
pelo contexto de cada paciente. A tematização destas questões, assim como o treinamento clínico prático sob princípios da PCC, deve fazer parte da formação profissional. Mesmo intervenções educativas curtas (de poucas horas e, portanto mais facilmente incorporadas a currículos já estruturados) podem produzir resultados positivos nas habilidades e crenças sobre compartilhamento de decisões entre estudantes das profissões de saúde ${ }^{21}$.

Em relação às barreiras no nível dos pacientes, problemas cognitivos, de expressão, de memória e de autopercepção são as mais frequentemente relatadas ${ }^{22}$. Alguns podem não compreender completamente as suas dificuldades e ter percepções pouco realistas de seu prognóstico. Isso suscita algumas questões. Quem é o cliente da PCC? Todo cliente tem condições de tomar decisões sobre seus cuidados? $\mathrm{O}$ que fazer, por exemplo, quando um idoso acamado e deprimido não deseja realizar quaisquer atividades propostas pelo terapeuta? Uma primeira resposta amplia o conceito de cliente para incluir familiares e cuidadores, que podem e desejam participar das decisões terapêuticas ${ }^{6}$. Outra resposta envolve o reconhecimento de que alguns pacientes desejam e podem articular suas necessidades e direcionar as decisões, outros nem sempre estão em posição de assumir tal poder e precisam ser direcionados. Os pacientes tem o direito de assumir responsabilidade, assim como também tem o direito de não assumir responsabilidade. A empatia para discernir entre situações é fundamental para a PCC.

Finalmente, as barreiras no nível institucional são de grande importância, pois fatores estruturais podem servir como forças modeladores para as maneiras de agir, pensar e sentir dos indivíduos - terapeutas e pacientes. Barreiras comuns incluem limites padronizados (e não individualizados) no número de sessões terapêuticas; escopo reduzido de serviços oferecidos, inviabilizando, por exemplo, visitas nos domicílios, escolas e locais de

\section{REFERÊNCIAS}

1. Rosewilliam S, Roskell CA, Pandyan A. A systematic review and synthesis of the quantitative and qualitative evidence behind patient-centred goal setting in stroke rehabilitation. Clin Rehabil. 2011;25(6):501-14. doi: 10.1177/0269215510394467.

2. Campos GWS. Saúde pública esaúde coletiva: campoe núcleo de saberes e práticas. Ciên Saude Coletiva. 2000;5(2):21930. doi: 10.1590/S1413-81232000000200002. trabalho e lazer; a impossibilidade de clínicos de diferentes profissionais trabalharem juntos para implantar projetos terapêuticos individualizados, mesmo que estes incluam técnicas e recursos normalmente vistos como fora do rol usual da sua profissão. A implantação da PCC exige mudança na cultura das organizações e das profissões de saúde. Há diversos instrumentos de avaliação que podem ser usados para caracterizar o grau de "centralidade" no cliente, tanto no nível institucional, quanto no nível dos profissionais e dos pacientes individualmente ${ }^{21,23-25}$. Por último, a formação de profissionais envolve investimentos financeiros, mas ao longo do tempo, os benefícios podem ultrapassar os custos ${ }^{19}$.

\section{CONSIDERAÇÕES FINAIS}

Este trabalho forneceu uma revisão narrativa dos fundamentos da PCC, instrumentos que podem ser usados para sua implementação, efeitos já documentados, assim como as principais dificuldades e benefícios da sua adoção na reabilitação. É preciso ressaltar que a PCC não se resume a quaisquer procedimentos, $\mathrm{e}$, portanto não pode ser definida pela adoção de instrumentos ou técnicas específicas de avaliação ou tratamento. Ao invés disso, a PCC se caracteriza por um conjunto de atitudes e valores individuais e institucionais em relação a todo o processo clínico. Tais atitudes e valores incluem a consideração do paciente como igual dentro da equipe de saúde, a valorização da comunicação - o que envolve escuta e educação dos pacientes - e a abertura para que os pacientes participem das decisões tanto quanto desejarem e façam suas próprias escolhas, adequadas ao seu contexto particular de vida. O conhecimento da PCC é fundamental para a missão profissional da terapia ocupacional e da fisioterapia de atender as demandas dos pacientes e oferecer cuidados com qualidade.

3. Law M, Baptiste S, Mills J. Client-centred practice: what does it mean and does it make a difference? Can J Occup Ther. 1995;62(5):250-57. doi: 10.1177/000841749506200504.

4. Sumsion T, Smyth G. Barriers to client-centredness and their resolution. Can J Occup Ther. 2001;67(1):15-21. doi: $10.1177 / 000841740006700104$.

5. Allen DD, Talavera C, Baxter S, Topp K. Gaps between patients' reported current and preferred abilities versus 
clinicians' emphases during an episode of care: any agreement? Qual Life Res. 2015;24(5):1137-43. doi: 10.1007/s11136-014-0888-0.

6. Law M, Baptiste S, Carswell A, McColl MA, Polatajko H, Pollock N. Medida canadense de desempenho ocupacional (COPM). Trad. Lívia de Castro Magalhães, Lilian Vieira Magalhães e Ana Amélia Cardoso. Belo Horizonte: Editora UFMG; 2009.

7. Bovend'Eerdt TJ, Botell RE, Wade DT. Writing SMART rehabilitation goals and achieving goal attainment scaling: a practical guide. Clin Rehabil. 2009;23(4):352-61. doi: 10.1177/0269215508101741.

8. Horn KK, Jennings S, Richardson G, van Vliet D, Hefford C, Abbott JH. The patient-specific functional scale: psychometrics, clinimetrics, and application as a clinical outcome measure. J Orthop Sports Phys Ther. 2012;42(1):30-D17. doi: 10.2519/jospt.2012.3727.

9. ÅsenlöfP, Siljebäck K. The patient goal priority questionnaire is moderately reproducible in people with persistent musculoskeletal pain. Phys Ther. 2009;89(11):1226-34. doi: 10.2522/ptj.20090030.

10. Doig E, Fleming J, Cornwell PL, Kuipers P. Qualitative exploration of a client-centered, goal-directed approach to community-based occupational therapy for adults with traumatic brain injury. Am J Occup Ther. 2009;63(5):55968. doi: 10.5014/ajot.63.5.559.

11. Young CA, Manmathan GP, Ward JC. Perceptions of goal setting in a neurological rehabilitation unit: a qualitative study of patients, carers and staff. J Rehabil Med. 2008;40(3):1904. doi: 10.2340/16501977-0147.

12. Webb PM, Glueckauf RL. The effects of direct involvement in goal setting on rehabilitation outcome for persons with traumatic brain injuries. Rehabil Psychol. 1994;39(3):179. doi: $10.1037 / \mathrm{h} 0080321$.

13. Arnetz JE, Almin I, Bergström K, Franzen Y, Nilsson H. Active patient involvement in the establishment of physical therapy goals: effects on treatment outcome and quality of care. Adv Physiother.. 2004;6(2):50-69. doi: 10.1080/14038190310017147.

14. Gagné DE, Hoppes S. The effects of collaborative goalfocused occupational therapy on self-care skills: a pilot study. Am J Occup Ther. 2003;57(2):215-9. doi: 10.5014/ ajot.57.2.215

15. Bassett SF, Petrie KJ. The effect of treatment goals on patient compliance with physiotherapy exercise programmes. Physiotherapy. 1999;85(3):130-7. doi: 10.1016/S00319406(05)65693-3.
16. Ostelo RWJG, de Vet HCW, Vlaeyen JW, et al. Behavioral graded activity following first-time lumbar disc surgery: 1-Year Results of a Randomized Clinical Trial. Spine. 2003;28(16):1757-65. doi: 10.1097/01. BRS.0000083317.62258.E6.

17. Eyssen IC, Steultjens MP, De Groot V, Steultjens EM, Knol DL, Polman CH, Dekker J. A cluster randomised controlled trial on the efficacy of client-centred occupational therapy in multiple sclerosis: good process, poor outcome. Disabil Rehabil. 2013;35(19):1636-46. doi: 10.3109/09638288.2012.748845.

18. Wilkins S, PollockN, Rochon S, Law M. Implementing clientcentred practice: why is it so difficult to do? Can J Occup Ther. 2011;68(2):70-9. doi: 10.1177/000841740106800203.

19. Mudge S, Stretton C, Kayes N. Are physiotherapists comfortable with person-centred practice? An autoethnographic insight. Disabil Rehabil. 2014;36(6):45763. doi: 10.3109/09638288.2013.797515.

20. Litchfield R, MacDougall C. Professional issues for physiotherapists in family-centred and community-based settings. Austr J Physiother. 2002;48(2):105-12. doi: 10.1016/S0004-9514(14)60204-X

21. Hoffmann TC, Bennett S, Tomsett C, Del Mar C. Brief training of student clinicians in shared decision making: a single-blind randomized controlled trial. J Gen Intern Med. 2014;29(6):844-9. doi: 10.1007/s11606-014-2765-5.

22. Kuipers P, Carlson G, Bailey S, Sharma A. A preliminary exploration of goal-setting in community-based rehabilitation for people with brain impairment. Brain Impairment. 2004;5(1):30-41. doi: 10.1375/brim.5.1.30.35404.

23. Shaw WS, Woiszwillo MJ, Krupat E. Further validation of the Patient-Practitioner Orientation Scale (PPOS) from recorded visits for back pain. Patient Educ Counseling. 2012;89(2):288-91. doi: 10.1016/j.pec.2012.07.017.

24. Dwamena F, Holmes-Rovner M, Gaulden CM, Jorgenson S, Sadigh G, Sikorskii A, Lewin S, Smith RC, Coffey J, Olomu A, Beasley M. Interventions for providers to promote a patient-centred approach in clinical consultations. Cochrane Database System Rev. 2012;12. doi: 10.1002/14651858. CD003267.pub2.

25. Dyke $\mathrm{P}$, Buttigieg $\mathrm{P}$, Blackmore A. M, Ghose A. Use of the Measure of Process of Care for families (MPOC-56) and service providers (MPOC-SP) to evaluate family centered services in a paediatric disability setting. Child Care Health Dev. 2006;32(2):167-76. doi: 10.1111/j.1365-2214.2006.00604.x. 